\title{
Impact of butyrate on PKM2 and HSP90 $\beta$ expression in human colon tissues of different transformation stages: a comparison of gene and protein data
}

\author{
Franziska Jahns · Anne Wilhelm • Karl Otto Greulich • \\ Henning Mothes · Mariya Radeva • \\ Anja Wölfert • Michael Glei
}

Received: 13 July 2011/ Accepted: 30 September 2011/Published online: 19 October 2011

(C) Springer-Verlag 2011

\begin{abstract}
Due to protection of oncogenic proteins from degradation and enhancement of glycolytic phosphometabolites for synthetic processes, respectively, heat shock protein 90 (HSP90) and pyruvate kinase type M2 (PKM2) are important proteins for tumor growth. The present study was undertaken to investigate the susceptibility of both proteins and their encoding genes to the chemopreventive agent butyrate in human colon cells. Matched tissue of different transformation stages derived from 20 individual colon cancer patients was used for the experiments. The results of quantitative real-time PCR revealed a moderate increase of $H S P 90 \beta$ and $P K M 2$ mRNA in colon tumors $(P<0.01)$ compared to normal tissues without relation to clinical parameters. The expression pattern could be confirmed for PKM2 protein by Western blot but not for HSP90 $\beta$. During culturing with butyrate, the amount of $P K M 2$ transcripts decreased in all three tissue types with the strongest effects observed in tumors (median fold decrease $45 \%, P<0.05)$. The protein data have not
\end{abstract}

F. Jahns $(\bowtie) \cdot$ A. Wilhelm $\cdot$ M. Glei

Department of Nutritional Toxicology, Institute of Nutrition,

Friedrich-Schiller-University Jena, Jena, Germany

e-mail: franziskajahns@aol.com

F. Jahns $\cdot$ A. Wilhelm $\cdot$ K. O. Greulich $\cdot$ M. Radeva Department of Single Cell and Single Molecule Techniques, Leibniz Institute of Age Research-Fritz Lipmann Institute, Jena, Germany

H. Mothes

Department of General, Visceral and Vascular Surgery, University Hospital, Friedrich-Schiller-University Jena, Jena, Germany

A. Wölfert

Institute of Pathology, Friedrich-Schiller-University Jena,

Jena, Germany reflected this influence supposing a more gradual degradation rate due to a longer half-life of PKM2. In contrast, the mRNA expression of $H S P 90 \beta$ in normal tissue was found 1.38 -fold increased by butyrate $(P<0.05)$, but not the corresponding protein level. HSP90 $\beta$ expression in adenomas and tumors remained generally insensitive. Only in malignant tissue, however, a significant correlation was found between the individual effects observed on gene and protein expression level. In conclusion, the present study identified PKM2 as a potential direct target of butyrate in neoplastic colon tissue, whereas HSP90 $\beta$ is none of it.

Keywords PKM2 - HSP90 - Butyrate - Colon cancer . Dietary fiber $\cdot$ Chemoprevention

$\begin{array}{ll}\text { Abbreviations } \\ \text { PKM2 } & \text { Pyruvate kinase type M2 } \\ \text { HSP90 } \beta & \text { Heat shock protein 90 beta } \\ \text { SCFA } & \text { Short chain fatty acids } \\ \text { HBSS } & \text { Hank's balanced salt solution } \\ \text { mRNA } & \text { Messenger RNA } \\ \text { cDNA } & \text { Complementary DNA } \\ \text { qPCR } & \text { Quantitative real-time polymerase chain } \\ & \begin{array}{l}\text { reaction } \\ \text { GUS }\end{array} \\ \text { HDAC } & \text { Histocuronidase } \\ \end{array}$

Introduction

Heat shock protein 90 (HSP90) and pyruvate kinase type M2 (PKM2) are essential for tumor growth and cancer metabolism and therefore, represent promising targets in the treatment of cancer (Christofk et al. 2008a; Neckers 
2007). HSP90 works as a molecular chaperone required physiologically for the maintenance and function of a wide range of proteins as well as mutated and aberrantly expressed proteins in cancer cells (Neckers 2007). These include transcription factors, protein kinases, and a large number of structurally unrelated proteins (e.g., steroid hormone receptors, Bcr-Abl, c-src, ErbB2, p53, cyclindependent kinases, telomerase, Akt, HIF-1 $\alpha$ ) (Pearl et al. 2008; Picard 2011). Stabilization of these client proteins that are often overexpressed in tumors prevents their degradation by the proteasome and consequently contributes to tumor development and cell survival (Brown et al. 2007). Blocking of HSP90 was shown to result in reduced invasiveness in vitro and in decreased tumor cell proliferation, vascularization as well as an improved efficacy of conventional therapy strategies in vivo (Moser et al. 2007). Further, HSP90 expressed by cancer cells protects against apoptosis (Rashmi et al. 2003), down-regulates tumorsuppressing signals such as TP53 (Lin et al. 2008) and enables replicative immortality through enhanced telomerase assembly (Akalin et al. 2001). HSP90 function is thereby implicated in the establishment of each of the hallmarks of cancer (Neckers 2007) that were first proposed and recently extended by Hanahan and Weinberg (2011). In addition to its client proteins, HSP90 itself was overexpressed in a variety of tumors, e.g., skin (Becker et al. 2004), prostate (Elmore et al. 2008), breast (Diehl et al. 2009), and colon (Milicevic et al. 2008). The function of HSP90 in tumorigenesis led to considerable interest in the chaperone as a target in cancer therapy. Inhibition of HSP90 is associated with the potential of down-regulating multiple oncogenic signaling pathways in which the various client proteins of HSP90 are involved. Classical inhibitors like geldanamycin and its derivates as well as synthetic molecules block the ATPase-coupled chaperone cycle and consign bound client proteins for ubiquitination and proteosomal degradation (Pearl et al. 2008). Targeting molecules that are involved in the regulation of HSP90, as for example histone deacetylase (HDAC) 6, is an alternative approach to inhibit chaperone activity (Pearl et al. 2008) and was, inter alia, demonstrated for genistein (Basak et al. 2008). Quercitin (Aalinkeel et al. 2008) and epigallocatechin-3-gallate (Yin et al. 2009) are further nutrition-related compounds with HSP90-inhibiting potential.

Besides deregulated control of cell proliferation, reprogramming of energy metabolism is essential for tumor growth and considered as an emerging hallmark of cancer cells (Hanahan and Weinberg 2011). Genes of glycolysis are involved in this adjustment, whereas PKM2 plays a key role. The enzyme represents one of 4 pyruvate kinase isoforms catalyzing the conversion of phosphoenolpyruvate to pyruvate during glycolysis. The isoform is typical for proliferating cells, such as in fetal and adult tissues, and for tumor cells. Malignant tissues express predominantly a dimeric, inactive form of PKM2 that is induced by direct interaction with oncoproteins or phosphotyrosine binding (Christofk et al. 2008b; Mazurek et al. 2005; Zwerschke et al. 1999). Catalytic inactive PKM2 is necessary for the metabolic switch from oxidative phosphorylation to aerobic glycolysis and proliferation of cells (Christofk et al. 2008b; Hitosugi et al. 2009). Attenuated PKM2 activity leads to increased lactate production and reduced oxygen consumption (Christofk et al. 2008a) that are typical features of the cancer cell's metabolism. Consequently, proliferation of tumor cells is promoted by a temporary accumulation of upstream phosphometabolites. These are becoming available as precursors for anabolic processes, such as DNA, amino acid, and phospholipid synthesis (Christofk et al. 2008b; Mazurek et al. 2005). Its involvement in carcinogenesis marks PKM2 as a target for the development and/or identification of novel cancer-related drugs as well. An inhibition of PKM2 has been shown to be accompanied by decelerated tumor cell proliferation and induced apoptosis as demonstrated by Spoden et al. (2008) and Guo et al. (2011) using specific PKM2 aptamers and RNA interference, respectively. So far, only indirect evidence is given regarding a regulation by bioactive food agents (Kim and Milner 2011). Components like luteolin (Byun et al. 2010), delphinidin (Fridrich et al. 2008), or butyrate (Hirsch et al. 2006) were reported to inhibit oncogenic tyrosine kinases that are known to participate in the regulation of PKM2 activity (Hitosugi et al. 2009).

The short chain fatty acid (SCFA) butyrate results, along with other products, from the colonic fermentation of dietary fibers (soluble) which belong to the most studied food ingredients in relation to colon cancer chemoprevention and are associated with a reduced risk for the disease (Bingham et al. 2003; Scharlau et al. 2009; Wong et al. 2006). Among the most abundant SCFA, butyrate represents about $20 \%$ and reaches absolute concentrations of $11-25 \mathrm{mM}$ in human feces, whereas concentrations in peripheral venous blood are even lower (Hamer et al. 2008; Wolever and Chiasson 2000). Compared to acetate or propionate, the $C_{4}$ carbonic acid is best characterized and linked to numerous anti-cancer effects in vitro and in vivo, e.g., induction of apoptosis, inhibition of proliferation, and modulation of various gene families (Borowicki et al. 2011; Pool-Zobel et al. 2005; Zhang et al. 2010). Therefore, the present study focused on butyrate and its impact on gene expression of HSP90 $\beta$ and PKM2 as well as their analog proteins in malignant and non-malignant (normal and adenoma) colon tissues derived from individual donors. Among the different types of tissue, adenoma is most interesting to study but difficult to obtain. Most tumors arise from these benign lesions, although only a small percentage of adenomas is 
going to progress to malignancy (Peipins and Sandler 1994). Among the 4 members of the HSP90 family, HSP90 $\beta$ was focused due to its overexpression in colon cancer as shown in own previous experiments (Radeva 2009). The outcomes of the study shall extend the few available data regarding the butyrate sensitivity of HSP90 and PKM2 and maybe identify a novel natural agent inhibiting both tumor markers.

\section{Materials and methods}

Tissue specimens

Colon tumor tissue and adjacent normal-appearing tissue were consecutively and unselectively obtained at the time of surgery from 20 patients undergoing colon surgical resection on schedule at the University Hospital of Jena. Normal colon samples were removed at a distance of $20-50 \mathrm{~cm}$ from the tumor site. Five out of the 20 patients had an additional or sole adenoma which was discovered accidently during surgery and also included in the investigations. Eight men and twelve women aged between 41 and 92 years attended the study. None of them has received chemotherapy or radiation prior to surgery. All tissue samples were checked and classified during histopathological examination by an experienced pathologist (Table 1). Normal tissues showed no signs of malignancy and inflammation and adenoma samples were proven to be benign. The tissue samples were stored in Hank's balanced salt solution (HBSS; $8.0 \mathrm{~g} / 1 \mathrm{NaCl} ; 0.4 \mathrm{~g} / 1 \mathrm{KCl} ; 0.06 \mathrm{~g} / \mathrm{l}$ $\mathrm{Na}_{2} \mathrm{HPO}_{4} \times 2 \quad \mathrm{H}_{2} \mathrm{O} ; 0.06 \mathrm{~g} / 1 \quad \mathrm{~K}_{2} \mathrm{HPO}_{4} ; 1 \mathrm{~g} / \mathrm{l}$ glucose; $0.35 \mathrm{~g} / \mathrm{l} \mathrm{NaHCO}_{3} ; 4.8 \mathrm{~g} / \mathrm{l}$ HEPES; $\left.\mathrm{pH} 7.2\right)$ on ice and prepared immediately as previously reported (Jahns et al. 2011). The resulting tissue strips of normal, adenoma, and tumor samples were either frozen in liquid nitrogen alone or submerged in RNA later (Qiagen, Hilden, Germany) and stored at $-80^{\circ} \mathrm{C}$ until cytosol or RNA was being extracted.

Treatment of colon tissue with butyrate

Simultaneously, strips from all 3 tissue types were randomly distributed to different wells which were used for the various experiments later on. Treatment with the same batch of an intestinally relevant dose of butyrate $(10 \mathrm{mM})$ (Hamer et al. 2008) for $12 \mathrm{~h}$ was performed in parallel, subsequently (Jahns et al. 2011). Primary cell culture medium (solvent for butyrate) was used as a control in the experiments and consisted of minimal essential medium with Earle's salts enriched with $20 \%$ fetal calf serum, $2 \mathrm{mM}$ glutamine, $100 \mu \mathrm{g} / \mathrm{ml}$ gentamycin, $2.5 \mu \mathrm{g} / \mathrm{ml}$ fungizone, $10 \mathrm{ng} / \mathrm{ml}$ epidermal growth factor, $5 \mu \mathrm{g} / \mathrm{ml}$ insulin,
$5 \mu \mathrm{g} / \mathrm{ml}$ transferrin, and $5 \mathrm{ng} / \mathrm{ml}$ sodium selenite according to Rogler et al. (1998). After 12-h incubation, the tissue strips used for protein analyses were washed in HBSS and frozen in liquid nitrogen. Samples for gene expression studies were additionally submerged in RNA later. Storage occurred equivalent to the other tissue specimens. According to Sauer et al. (2007), 12 h was the maximum duration of treatment for primary colon tissue to get enough viable cells and intact RNA.

Isolation of RNA and reverse transcription into complementary DNA (cDNA)

RNA isolation and cDNA synthesis were performed as previously described (Jahns et al. 2011). In short, total RNA was isolated from the homogenized tissue samples by using the RNeasy Plus Mini Kit (Qiagen) according to the manufacturer's protocol followed by spectrophotometrically quantification with the NanoDrop ${ }^{\circledR}$ ND-1000 (NanoDrop Technologies, Wilmington, DE). Integrity of the RNA was determined with the Agilent 2100 Bioanalyzer (Agilent Technologies, Palo Alto, CA). Only RNA of sufficient quality (RNA integrity number $>5$ ) (Fleige et al. 2006) was reverse transcribed using the SuperScript II First Strand cDNA Synthesis System (Invitrogen, Darmstadt, Germany). Due to variation of quantity of extracted total RNA, the amount used as a template varied with each patient from 100 to $2,500 \mathrm{ng}$.

Quantitative real-time polymerase chain reaction (qPCR)

Quantitative PCR conditions were already described by Jahns et al. (2011). Briefly, $2 \mu \mathrm{l}$ cDNA prepared from different starting concentrations of total RNA were amplified in three steps by using iQ SYBR Green Supermix (Bio-Rad, Munich, Germany) and 10 pmol gene-specific primers: $H S P 90 \beta$ forward, 5'-CGTTGCTCACTATTACG TATAATCCT- $3^{\prime}$ and reverse, $5^{\prime}$-CGAATCTTGTCCAAG GCATC-3'; PKM2 forward, 5'-TCCGGATCTCTTCGTC TTTG-3' and reverse, 5'-TGGGTCTGAATGAAGGCA GT-3'; $\beta$-actin forward, 5'-AGAGCCTCGCCTTTGCCG AT- $3^{\prime}$ and reverse, $5^{\prime}$-CCCACGATGGAGGGGAAGAC-3'; $\beta$-glucuronidase (GUS) forward, 5'-TGCAGGTGATGGAA GAAGTG- $3^{\prime}$ and reverse, 5'-TTGCTCACAAAGGTCACA GG-3'.

The expression of the targets was normalized to the geometric average (Vandesompele et al. 2002) of two reference genes ( $\beta$-actin, GUS) based on the equation of Pfaffl (2001) involving efficiency $(E)$ and quantification cycle $\left(C_{\mathrm{q}}\right)$. Since the reaction efficiencies of all primer pairs were close to $100 \%$, E was set to 2 . 
Table 1 Clinicopathological data of colorectal cancer patients analyzed in this study

\begin{tabular}{ll}
\hline Patient and tumor characteristics & Number of patients \\
\hline Mean age (years) \pm SD & $70.5 \pm 13.2$ \\
Gender & 12 females, 8 males \\
Tumor stage & \\
I & 2 \\
II & 6 \\
III & 8 \\
IV & 3 \\
Tumor grading & \\
1 & - \\
2 & 10 \\
3 & 7 \\
4 & 1 \\
Unknown & 1
\end{tabular}

One of the 20 donors was only found with adenoma. Tumor stage was assessed according to the Union for International Cancer Control classification

Relative mRNA expression $=\frac{\sqrt{E^{C_{q}(\beta-\text { actin })} \times E^{C_{q}(\mathrm{GUS})}}}{E^{C_{q} \text { target }}}$.

Cytosol extraction and Western blot analysis

Butyrate-treated $(10 \mathrm{mM})$ and non-treated pieces of tissue samples (normal, adenoma, and tumor) of the same set of patients that was already used for mRNA expression analysis were homogenized in cold lysis buffer $(50 \mathrm{mM}$ $\mathrm{KH}_{2} \mathrm{PO}_{4} ; 1 \mathrm{mM} \mathrm{Na} 2$ EDTA; $0.1 \%$ Triton X-100 and $1 \mathrm{mM}$ Pefabloc; pH 7) with the Polytron homogenizer 2100 (Kinematica AG, Lucerne, Switzerland) and centrifuged $\left(16,000 \mathrm{~g}, 10 \mathrm{~min}, 4^{\circ} \mathrm{C}\right)$. Total protein contents were determined according to Bradford (1976). For Western blot analysis, $10-30 \mu \mathrm{g}$ of total protein was diluted with $5 \times$ concentrated loading buffer $(250 \mathrm{mM}$ Tris- $\mathrm{HCl}(1 \mathrm{M}) \mathrm{pH}$ $6.8 ; 10 \%$ sodium dodecyl sulfate; $50 \%$ glycerol; $0.1 \%$ bromphenolblue and $0.5 \mathrm{M}$ dithiothreitol), separated by sodium dodecyl sulfate polyacrylamide gel electrophoresis (stacking gel: 4\%; separating gel: 12\%; Bio-Rad) and transferred to a nitrocellulose membrane (Whatman, Florham Park, NJ). Subsequently, the membrane was blocked with 5\% nonfat dried milk powder $(1 \mathrm{~h}$; AppliChem, Darmstadt, Germany) and incubated with the following primary and secondary antibodies: HSP90 $\beta$ (Zymed Laboratories, San Francisco, CA), PKM2 (Cell Signaling Technology, Danvers, MA), $\beta$-actin (Sigma-Aldrich, Steinheim, Germany), Polyclonal Rabbit Anti-Mouse IgG/HRP (Dako, Hamburg, Germany), and Polyclonal Goat Anti-Rabbit IgG/HRP (Dako). Detection and evaluation of protein bands were performed as previously described (Jahns et al. 2011).
Statistical evaluation

Statistical analysis was performed with the GraphPad Prism Software 5 (GraphPad Software, San Diego, CA). By using the nonparametric Wilcoxon matched pairs test or the Kruskal-Wallis test, two or multiple groups, respectively, were examined for significant differences. The correlation between two parameters was evaluated by the Spearman's rank correlation test. Gender differences were identified by the Mann-Whitney test. All results reached significance when $P<0.05$.

\section{Results}

$P K M 2$ and $H S P 90 \beta$ mRNA expression are moderately elevated in tumor tissues of colorectal cancer patients

The tumor markers $P K M 2$ and $H S P 90 \beta$ were found to be moderately overexpressed in colon cancer tissue when compared to the related normal counterparts $(P<0.01$, Fig. 1). PKM2 showed a median increase of 1.41 -fold (Fig. 1a), whereas $H S P 90 \beta$ was median 1.81-fold up-regulated in the malignant tissue (Fig. 1b). The adenoma samples investigated showed also enhanced mRNA levels of both genes (median fold change PKM2 and HSP9OB: 2.13 and 2.14 , respectively) when compared to the respective normal tissue, but only $P K M 2$ reached almost statistical significance ( $P=0.06$, Fig. 1$)$.

Correlation analyses according to Spearman revealed no connection between PKM 2 or HSP $90 \beta$ mRNA expression and clinical parameters such as tumor stage and gender (data not shown). Nevertheless, a tendency toward increased PKM2 mRNA levels in undifferentiated colon cells (determined by the tumor grading: from G1 to G4) has been noted ( $r=0.43, P=0.07$, Fig. 2a). Additionally, $P K M 2$ expression seems to change in colon tissue from elderly humans $(r=-0.43, P=0.06$, Fig. $2 b)$. Younger donors have been found with higher amounts of PKM2 transcripts in normal colon epithelium compared to older ones. No link between these parameters existed in tumor tissue (data not shown). Transcription of $H S P 90 \beta$ was independent of tumor grade and age (data not shown).

In vitro culturing of epithelial tissue strips induces expression changes of $P K M 2$ and $H S P 90 \beta$ mRNA

Culturing of normal and tumor colon tissues ex vivo (12 h) by using our primary cell culture medium significantly augmented $P K M 2$ and $H S P 90 \beta$ mRNA expression (Fig. 1a, b). Median increases ranged from 1.49- to 2.17fold when compared to the respective basal transcript levels. Benign adenoma samples reacted less sensitive to 
Fig. 1 Basal mRNA expression of $P K M 2$ (a) and $H S P 90 \beta$ (b) and its alteration after cell culturing in paired normal, adenoma, and tumor colon tissues. mRNA levels were quantified before $(0 \mathrm{~h}$, filled square minus) and after the treatment with medium $(12 \mathrm{~h}$, open circle minus) in a humidified incubator $\left(37^{\circ} \mathrm{C}, 5 \%\right.$ $\mathrm{CO}_{2}$ ) by using qPCR. Data are arranged by age of the patients (P). The small letter discriminates between male (m) and female (f). For better visualization, basal levels and culturing effects were each connected by a line. Twenty patients in total were investigated. Four of them were found with adenomas besides tumor and adjacent normal colon tissue and one was only discovered with adenoma and adjacent normal colon tissue a

PKM2

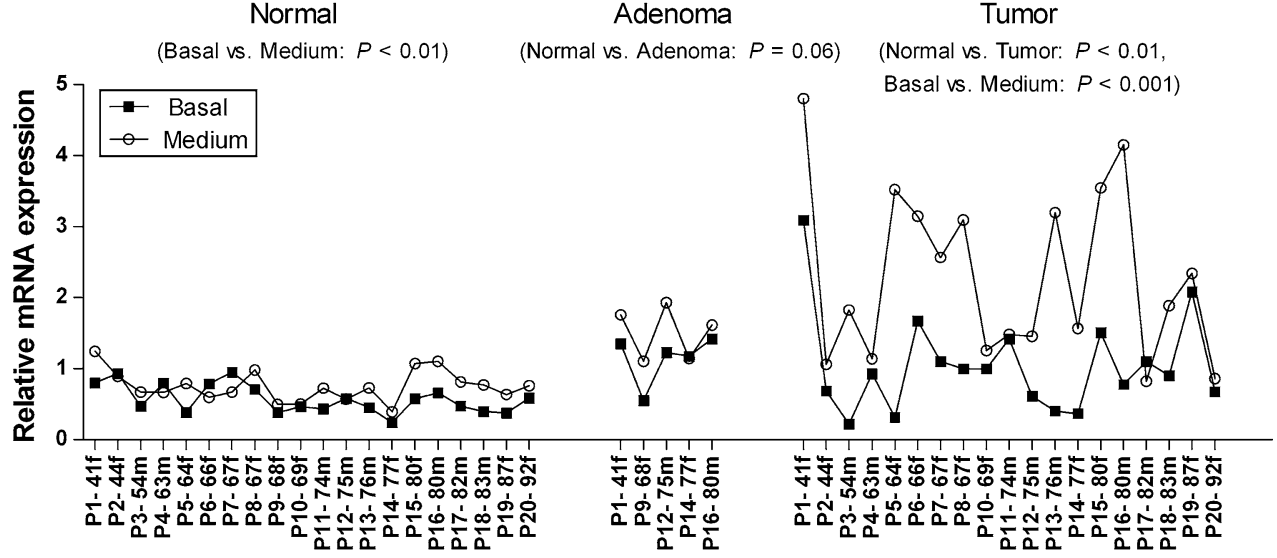

Patients
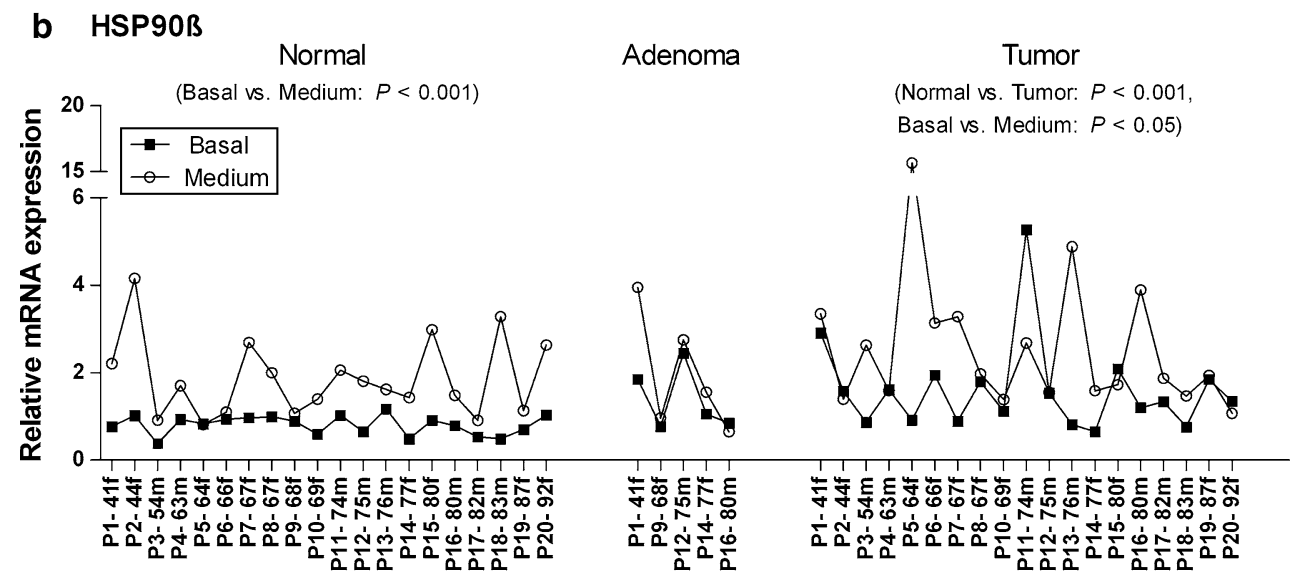

Patients the incubation. By showing median elevations of 1.24 (HSP90 $\beta$ )- and 1.30 (PKM2)-fold compared to the related untreated tissue, adenoma samples remained almost unaffected $(P>0.05$, Fig. 1a, b).

Butyrate affects $P K M 2$ and $H S P 90 \beta$ mRNA expression differently

To assess the effects of the SCFA, the expression of the target genes ascertained in the butyrate-treated tissues had to be compared to those of the respective medium controls. In regard to PKM2 and $H S P 90 \beta$, the tissues showed different susceptibility to butyrate.

The amount of PKM2 transcripts was decreased by butyrate in all 3 tissue types but only the result of tumor tissue reached statistical significance (Fig. 3a). In normal tissue, PKM2 mRNA level was predominantly slightly diminished (median fold change 0.81 ), whereas sensitivity to butyrate increased with advancing grade of transformation. Benign
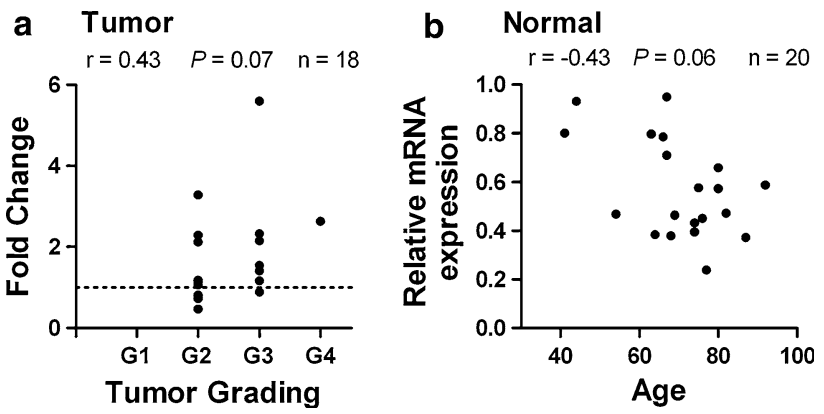

Fig. 2 Impact of clinical parameters on PKM2 gene expression in normal and malignant colon tissue. Total RNA from paired normal, adenoma, and tumor colon tissues (filled circle) was quantified by qPCR and compared to diverse clinical data by Spearman correlation analysis. Twenty patients in total were investigated, whereby only 4 of them had all three tissue types. Trends and significant outcomes are presented. a In cancer tissue, an up-regulation of PKM2 mRNA was observed with progress of dedifferentiation indicated by the tumor grading. The dotted line denotes no expression changes (fold change 1). b In normal colon tissue, $P K M 2$ mRNA expression went down with increasing age of patients 
adenoma samples showed a 0.69 -fold (median) reduction of PKM2 expression when compared to the related medium controls. In the corresponding tumor tissues, butyrate was able to suppress the medium-induced $P K M 2$ mRNA increase by median 0.55 -fold $(P<0.001)$. Fourteen out of nineteen patients possessing tumors showed a decreased transcript level of the glycolytic enzyme. Gender, age, tumor stage, or grading were not linked to the individual effect (data not shown). Besides these clinicopathological features, we tested further whether the initial PKM2 transcript amount could be accountable for the variations in each patient. According to the Spearman correlation test, we noted by trend that PKM2 mRNA expression was more effectively suppressed by butyrate in normal tissues showing a low basal status of $P K M 2$ transcripts compared to those with a high $P K M 2$ basal level ( $r=0.41, P=0.08$, data not shown). For adenoma tissue a similar correlation is suggested, but due to the limited number of donors $(n=5)$ no statistical significance was found (data not shown). In the malignant tissue, however, modification of PKM2 after treatment with butyrate was not linked to the respective basal mRNA level (data not shown).
Besides the effect of medium on $H S P 90 \beta$, its supplementation with butyrate additionally enhanced the mRNA expression of the stress gene by median 1.38 -fold in normal colon cells $(P<0.05$, Fig. 3b). However, in adenoma and tumor tissue, greater interindividual variations were observed in response to the $\mathrm{C}_{4}$ carbonic acid that reached no significant outcome (fold change adenoma and tumor: 0.72 and 1.09, respectively). Clinicopathological data (age, gender, tumor stage, and grading) or the initial transcript level did not account for the varying effects between individuals. Despite the $H S P 90 \beta$ mRNA rise in normal cells, the effect of butyrate was not significantly different from that in tumor tissue.

PKM2 and HSP90 $\beta$ protein expression are not targeted by butyrate

For detection of PKM2 and HSP90 $\beta$ proteins, identical treated sample material of the patients whose mRNA expression was previously analyzed has been used. Thereby, Western blot was only performed with tissue
Fig. 3 Impact of butyrate (filled triangle) on PKM2 (a) and HSP90 $\beta$ (b) mRNA expression in matched normal, adenoma, and tumor colon tissues. Relative $P K M 2$ and $H S P 90 \beta$ mRNA levels were determined after butyrate administration $(10 \mathrm{mM}, 12 \mathrm{~h})$ by qPCR and compared to the respective medium controls (open square) which were set to 1 (fold change). Results are listed by age of the patients $(\mathrm{P})$, the small letter discriminates between male (m) and female (f). Twenty patients in total were investigated. Four of them had available adenomas besides tumor and adjacent normal colon tissue and one was only found with adenoma and adjacent normal colon tissue

\section{a PKM2}
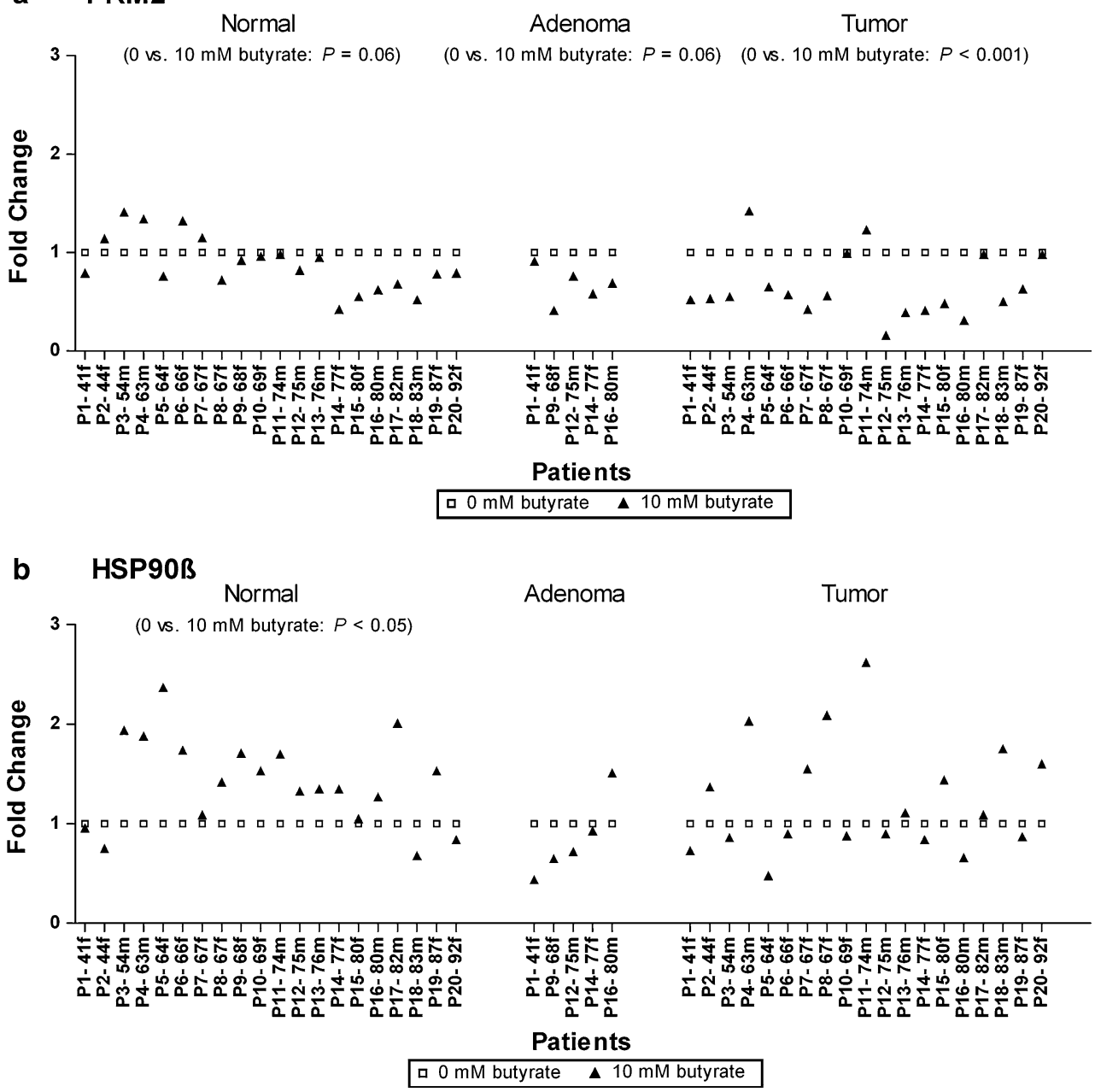


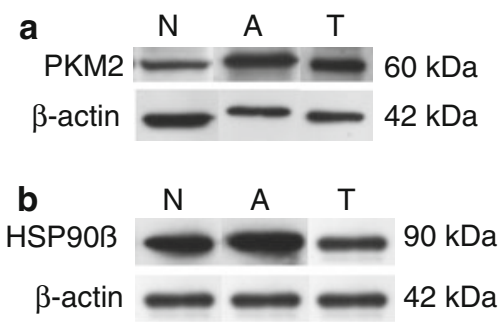

Fig. 4 Representative blots of PKM2 (a) and HSP90 $\beta$ (b) basal protein expression in matched normal $(\mathrm{N})$, adenoma $(\mathrm{A})$, and tumor (T) colon tissue. Cytosolic extracts from all three types of tissue were analyzed by Western blot using specific antibodies. $\beta$-actin served as internal loading control

specimens of selected patients mainly showing a modified mRNA expression after butyrate treatment.

According to its basal mRNA expression, PKM2 protein was median mostly present in benign adenoma samples (2.12-fold) followed by tumor specimens (median 1.24fold, $P<0.05$ ) when compared to the related normal counterparts (data not shown except example blot, Fig. 4a). However, a patient-wise comparison of the gene and protein expression data of PKM2 demonstrated no correlation between both parameters (adenoma: $r=0.1, P=0.95$, tumor: $r=0.30, P=0.43$, data not shown).

In contrast to PKM2, HSP90 $\beta$ partly showed a divergent expression pattern at the protein level as compared to its mRNA expression. In adenoma tissue, moderately elevated levels of HSP90 $\beta$ (median 1.59-fold, $P=0.125$ ) were detected, whereas its median expression in tumors remained almost unchanged (0.92-fold, data not shown except example blot, Fig. 4b). Again, gene and protein expression data were not congruent when comparing them in a patientwise manner (adenoma: $r=-0.8, P=0.33$, tumor: $r=$ $-0.05, P=0.88$, data not shown).

During culturing of the epithelial tissue strips the expression of PKM 2 and HSP90 $\beta$ protein remained relatively constant in all tissue types, ranging from median 0.71-fold decrease to 1.19-fold increase (data not shown).

After butyrate treatment, the expected decrease of PKM2 protein in tumors did not occur. Unlike the data from qPCR, protein expression of the glycolytic enzyme was not significantly modified by the SCFA in normal and malignant tissues (median fold changes normal and tumor: 1.02 and 1.03 , respectively, Fig. 5a, b). Further, no correlation existed between PKM2 mRNA and protein abundance of the 13 donors investigated (Fig. 5a, b). Only 20\% of them exhibited an almost conformity of both parameters (deviations of fold changes less than 25\%). Benign adenoma samples were observed with similar susceptibility to butyrate and showed median unaltered levels of PKM2 protein (0.97-fold, data not shown), in contrast to the gene expression data (median 0.69-fold).
In regard to HSP90 $\beta$, the up-regulation of its mRNA in normal colon cells after butyrate treatment was not reflected by the corresponding protein expression. HSP $90 \beta$ protein remained nearly unchanged in the presence of butyrate (median fold change $0.88, P=0.95$ ) as compared to its transcript amount (Fig. 5c). A correlation analysis of both parameters according to Spearman confirmed this issue $(r=0.13, P=0.68)$. However, for the malignant tissue a contrary result was observed which maybe points to varying transcriptional mechanisms in normal and tumor colon tissue. In agreement with qPCR experiments, the individual effects of butyrate corresponded to those observed by Western blot. A subsequent correlation analysis confirmed this link by showing a significant correlation between mRNA and protein abundance of HSP90 $\beta$ $(r=0.68, P<0.05$, Fig. 5 d). However, HSP90 $\beta$ protein was generally not altered in tumor tissue by butyrate (median fold change 0.93).

In pre-neoplastic tissue, HSP90 $\beta$ was found similar sensitive to inhibition by butyrate (median fold change 0.84 ) as normal tissue. Although gene expression is reflected by the protein level of HSP90 $\beta$ when comparing the median expression values, no correlation could be found by comparing the individual data so far $(r=-0.50$, $P=0.39$, data not shown).

\section{Discussion}

Basal expression levels

In accordance with previous data from our own research and that of others (Koss et al. 2008), PKM2 was up-regulated in colon adenomas and tumors. But in contrast to these studies, our data were not related to tumor stage which might be due to different reasons (e.g., sample material, number of patients). Although a relative good agreement could be observed between the median gene and protein expression of the glycolytic enzyme in adenoma and tumor samples, no correlation was detected when the data were compared in a patient-wise manner. The same applies for HSP90 $\beta$. The discrepancy between mRNA and protein abundance in tumor tissue was already described by Radeva (2009) and Chen et al. (2002) for colon and lung, respectively. Research studies of this type are generally rare to be found. The inconsistencies in results may be originate from the use of different tissue pieces for the various analyses, cell-specific regulation of protein translation, or post-transcriptional mechanisms.

Furthermore, correlation tests revealed an age-related decrease of PKM2 mRNA expression in normal colon epithelium, which, however, could not be confirmed at protein level. Previous studies already reported a differential gene 
Fig. 5 Effects of butyrate on PKM2 and HSP90 $\beta$ protein in colon tissues of different transformation stages. a, c Representative blots of PKM2 and HSP90 $\beta$ protein in paired normal and tumor colon tissue after treatment with $10 \mathrm{mM}$ butyrate for $12 \mathrm{~h}$. Cytosolic extracts from the different tissue types have been analyzed by Western blot using specific antibodies. $\beta$-actin served as internal loading control. b, d Protein expression ratios (fold changes) of PKM2 and HSP90 $\beta$ in paired normal and tumor colon tissue after treatment with $10 \mathrm{mM}$ butyrate for $12 \mathrm{~h}$ compared to the respective gene expression ratios. Sample material from donors was simultaneously treated with butyrate $(12 \mathrm{~h})$ for subsequent mRNA (qPCR) and protein expression analysis (Western blot). Results are presented in a patient $(\mathrm{P})$-wise manner, sorted by age of the patients. The small letter further differentiates between males $(\mathrm{m})$ and females (f). The symbols (filled triangle, open square minus) denote the change in expression after butyrate treatment when compared to the medium control (fold change). No protein fold change could be determined for the tumor samples of patients P12 (PKM2) and P19 (HSP90 $\beta$ ). Ratios equal to 1 indicate no expression differences between butyrateand non-butyrate-treated (medium) tissues $(n=13)$

\section{PKM2}

a

\begin{tabular}{lll} 
Butyrate & $0 \quad 10$ & $\mathrm{mM}$ \\
\hline PKM2 & & $60 \mathrm{kDa}$ \\
$\beta$-actin & & $42 \mathrm{kDa}$
\end{tabular}

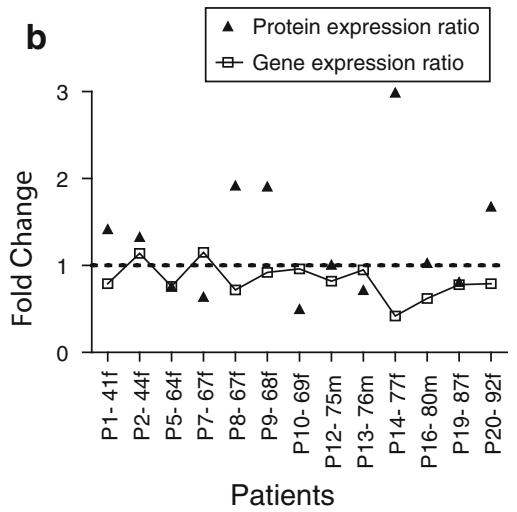

HSP90ß
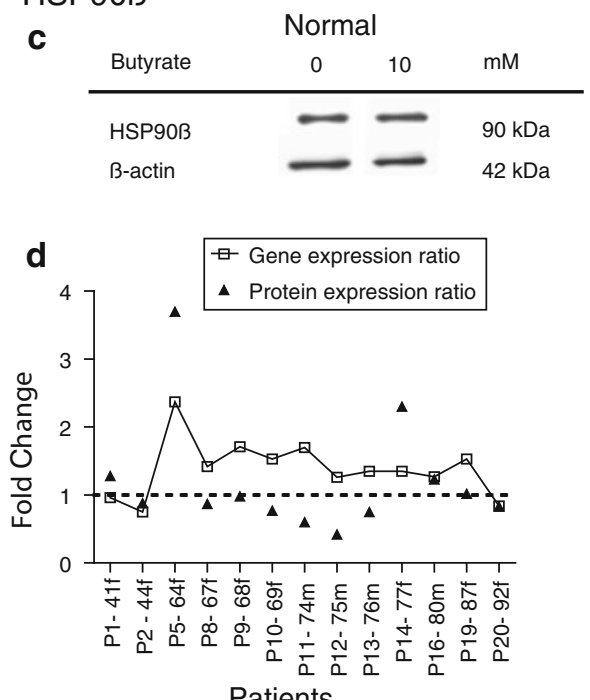

Patients

\begin{tabular}{lccc}
\multicolumn{4}{c}{ Tumor } \\
Butyrate & 0 & 10 & $\mathrm{mM}$ \\
\hline PKM2 & - & - & $60 \mathrm{kDa}$ \\
$\beta$-actin & - & $42 \mathrm{kDa}$
\end{tabular}

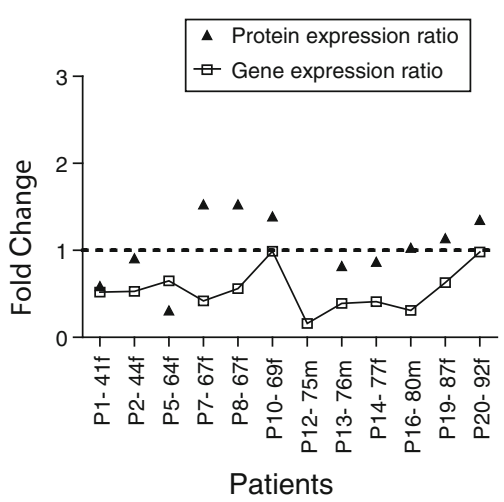

\begin{tabular}{lccc} 
& \multicolumn{2}{c}{ Tumor } & \\
Butyrate & 0 & 10 & $\mathrm{mM}$ \\
\hline HSP90B & & $90 \mathrm{kDa}$ \\
B-actin & & $42 \mathrm{kDa}$
\end{tabular}

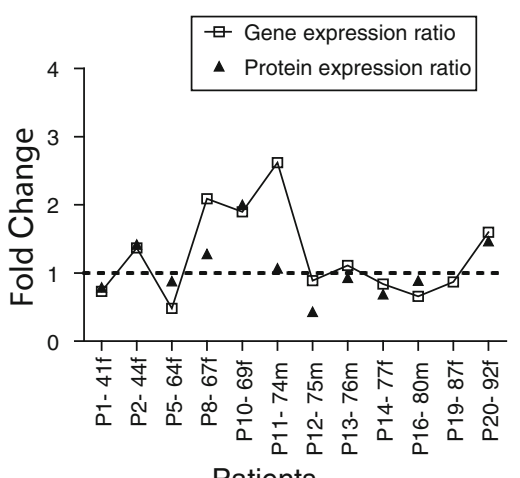

Patients and/or protein expression for a variety of genes in senescent tissues including those involved in glycolytic energy metabolism (Cho et al. 2003; Lee et al. 2001; O'Connell et al. 2007). Expression and activity of pyruvate kinase isoenzymes were found mainly up-regulated, as for example in liver (Cho et al. 2003) and fibroblasts (Zwerschke et al. 2003). For colon tissue, no change in the glycolytic flux was noted (Fleming and Gill 1997), indicating that no enzymes of this metabolic pathway have been modified. The present finding of down-regulated $P K M 2$ in colon cells from elderly humans is contrary to most other reports mentioned and its significance is yet not clear to date nor can be explained by the current literature.
Influence of cell culturing on expression levels

Modulation of gene expression during culturing was already observed in own previous experiments investigating cyclooxygenase-2 and osteopontin (Jahns et al. 2011) but in contrast to those, accumulation of PKM2 and $H S P 90 \beta$ mRNA was comparably low. PKM expression is physiologically regulated by hormonal (e.g., insulin, thyroid hormones) and nutritional influences (e.g., glucose, glutamine) (Yamada and Noguchi 1999) that are partly present in our primary cell culture medium. Decaux and colleagues demonstrated in primary rat hepatocytes expressing pyruvate kinase type L (PKL) that glucose and 
insulin are indispensable for stimulation and maintenance of PKL mRNA expression during culturing (Decaux et al. 1989). Similar findings were also made for PKM in adipocytes (Traxinger and Marshall 1992) and thymocytes (Greiner et al. 1994).

In case of HSP90 $\beta$, no direct evidence was found as reference to the induction of its mRNA expression by medium components. The environmental and nutritional changes that are rather supposed for its increase therefore necessitate the comparison of butyrate effects to those of a medium control.

Influence of butyrate on expression levels

Studies investigating directly the regulation of PKM2 expression by bioactive compounds are apparently lacking in current literature. The present one is the first that tested the influence of the gut fermentation product butyrate on the glycolytic enzyme. In contrast to clinical studies including a large number of participants, ours represents an intermediate step which allows studying of individual effects on a small cohort of patients. Previous studies of our group in regard to the proportion of viable cells demonstrated viabilities of primary colon epithelial cells of mean $76 \%$ and intact RNA after the $12-\mathrm{h}$ incubation period that were in an acceptable range for the performance of the experiments (Sauer et al. 2007). These results were shown with several individual donors. Although early apoptosis cannot be excluded at this time, other authors reported that induction of apoptosis and inactivation of survival pathways are blocked by preserving cell-cell contacts (Hofmann et al. 2007), as in the tissue strips.

The treatment of normal and tumor colon tissues with the SCFA caused a tissue-specific regulation of PKM2 mRNA. But the effects in tumors were not reflected by the corresponding protein of PKM2 gene. A similar result was also obtained for adenoma tissue when comparing the median fold changes. Post-transcriptional modifications of PKM2 which might explain the difference between mRNA and protein level are not described in the literature. Further, we cannot rule out that the down-regulation of PKM2 protein was not equivalent due to the half-life of the protein and occurs possibly at a later date. Detailed information on this point is currently not available according to our search. Depending on the isoenzyme and tissue, respectively, different half-lives of pyruvate kinases are reported that all take up to several hours (Jones and Mayer 1973; Poole and Bloxham 1982). By the current improvement of our primary colon cell culture, kinetic studies above $12 \mathrm{~h}$ will be possible and either confirm or confute this hypothesis, prospectively.

An inhibition of PKM2 would be preferable since it is accompanied by decelerated tumor cell proliferation, as demonstrated by Spoden et al. (2008). The mechanism by which butyrate is acting on PKM2 expression is currently unknown. It is conceivable that the SCFA might downregulate directly or indirectly $\mathrm{PKM} 2$ via its HDAC inhibitory potential. HDAC inhibitors, including butyrate, have been demonstrated to target oncogenic tyrosine kinases, such as Scr kinase (Hirsch et al. 2006), which are involved in the regulation of PKM2 activity (Christofk et al. 2008b). Those proteins post-translationally modify PKM2 through phosphorylation at specific tyrosine sites resulting in the disruption of the formation of the active PKM2 tetramer and consequently attenuation of catalytic activity (Hitosugi et al. 2009).

HSP90 $\beta$ revealed nearly unaffected in the presence of butyrate in all tissues when compared to the respective medium controls. The exposure of normal colon tissue to butyrate $(10 \mathrm{mM})$ has been initiated cellular stress responses as indicated by a slight but significant $H S P 90 \beta$ mRNA increase. Even if the protein level would be affected, the relevance of this alteration regarding colon carcinogenesis might crucially depend on the duration of induction. Additionally, the function of HSP90 in normal cells has to be taken into account. By protecting cells from apoptosis under stress conditions (e.g., heat shock) (Beere 2005), the increase of HSP90 $\beta$ is probably an effort of the cell to support its own survival during culturing ex vivo. Similar observations after butyrate treatment (3-5 mM) were also made by Cai et al. (2006) using HT29 colon adenocarcinoma cells.

In adenoma and tumor colon tissue, neither gene nor protein expression was significantly altered by butyrate. But in contrast to normal mucosa and adenomas, both parameters demonstrated a good conformity in tumor tissue independent of age of the patients. These results suggest a different regulation of HSP90 $\beta$ in the various tissues, whereby in tumors regulation is likely to occur at the transcriptional level.

Due to its involvement in carcinogenesis, HSP90 represents an interesting target in cancer therapy (Pearl et al. 2008). Besides known natural and synthetic agents, a number of different bioactive food components, such as quercitin (Aalinkeel et al. 2008), genistein (Basak et al. 2008), and epigallocatechin-3-gallate (Tran et al. 2010), were also identified as potent HSP90 inhibitors. Modulation of post-translational mechanisms is an important approach to interfere with the regulation of HSP90. The three major modifications are phosphorylation, acetylation, and S-nitrosylation (Trepel et al. 2010). With regard to the potential of butyrate as an HDAC inhibitor, HDAC 6 plays a predominant role in the regulation of HSP90 activity. HSP90 hyperacetylation that is induced by HDAC inhibitors or silencing of HDAC6 correlates with the disturbance of its chaperone function and destabilization of HSP90 client proteins (Bali et al. 2005; Kekatpure et al. 2009). Although the total protein level of HSP90 is not changed (as shown in our experiments) and solely the fraction of 
acetylated HSP90 increases in this process, previous investigations demonstrated no effect of butyrate on acetylation status and ATP binding of HSP90 (Bali et al. 2005). The missing alteration is due to the structure of the SCFA. Only hydroxamic acid derivates and seemingly phenolic components, like genistein, are able to inhibit HDAC6 activity, whereas butyrate is specifically blocking HDAC1 and 3 (Barlow et al. 2001; Marks and Xu 2009; Thangaraju et al. 2009). In a human tumor model of leukemia, a synergistic interaction has been observed by combining butyrate and the HSP90 inhibitor 17-allylamino-17-demethoxygeldanamycin (Rahmani et al. 2003).

Looking at the individual data, some donors have been found with increased HSP90 $\beta$ levels in tumor tissue after butyrate treatment. Increased expression of HSPs or the transcriptional regulator heat shock factor 1, respectively, is associated with decreased sensitivity of tumor cells to chemopreventive drugs, such as butyrate (Cai et al. 2006) or curcumin (Rashmi et al. 2003), by protecting those from apoptosis or differentiation inducing mechanisms. To assess the meaning of this increase and its consequences for the application of chemopreventive drugs, kinetic studies will prospectively clarify, if this increase is of transient or persistent nature.

In summary, PKM2 has been identified as a potential target of the chemopreventive agent butyrate in human colon tumors. Although HSP90 $\beta$ was not modified by the SCFA in malignant tissue in general, a group of patients was observed with increased levels of HSP90 $\beta$ after treatment. Further studies should clarify which conditions permit this reaction and its duration, as well as find out more details about the post-transcriptional and translational machinery of PKM2.

Acknowledgments The present study was supported by the German Research Foundation (DFG, PO 284/8-3). Additionally, we are grateful to Kerstin Kalmring-Raspe, Dr. Wiebke Schlörmann, and Nadja Jablonowski for technical assistance and their helpful encouragement. We also thank Misses Kornelia Haus who edited the language of the manuscript and all tissue donors for giving their informed consent and thereby allowing the performance of this study.

Ethical standards The study has been approved by the ethics committee of the University Hospital of Jena, Germany (no. 1601-08/ 05 ) and has therefore been performed in compliance with the Declaration of Helsinki and its later amendments. Prior to study participation, all patients gave their informed consent.

Conflicts of interest None.

\section{References}

Aalinkeel R, Bindukumar B, Reynolds JL, Sykes DE, Mahajan SD, Chadha KC, Schwartz SA (2008) The dietary bioflavonoid, quercetin, selectively induces apoptosis of prostate cancer cells by down-regulating the expression of heat shock protein 90 . Prostate 68:1773-1789

Akalin A, Elmore LW, Forsythe HL, Amaker BA, McCollum ED, Nelson PS, Ware JL, Holt SE (2001) A novel mechanism for chaperone-mediated telomerase regulation during prostate cancer progression. Cancer Res 61:4791-4796

Bali P et al (2005) Inhibition of histone deacetylase 6 acetylates and disrupts the chaperone function of heat shock protein 90: a novel basis for antileukemia activity of histone deacetylase inhibitors. J Biol Chem 280:26729-26734

Barlow AL, van Drunen CM, Johnson CA, Tweedie S, Bird A, Turner BM (2001) dSIR2 and dHDAC6: two novel, inhibitor-resistant deacetylases in Drosophila melanogaster. Exp Cell Res 265:90103

Basak S, Pookot D, Noonan EJ, Dahiya R (2008) Genistein downregulates androgen receptor by modulating HDAC6-Hsp90 chaperone function. Mol Cancer Ther 7:3195-3202

Becker B, Multhoff G, Farkas B, Wild PJ, Landthaler M, Stolz W, Vogt T (2004) Induction of Hsp90 protein expression in malignant melanomas and melanoma metastases. Exp Dermatol 13:27-32

Beere HM (2005) Death versus survival: functional interaction between the apoptotic and stress-inducible heat shock protein pathways. J Clin Invest 115:2633-2639

Bingham SA et al (2003) Dietary fibre in food and protection against colorectal cancer in the European Prospective Investigation into Cancer and Nutrition (EPIC): an observational study. Lancet 361:1496-1501

Borowicki A, Michelmann A, Stein K, Scharlau D, Scheu K, Obst U, Glei M (2011) Fermented wheat aleurone enriched with probiotic strains LGG and Bb12 modulates markers of tumor progression in human colon cells. Nutr Cancer 63:151-160

Bradford MM (1976) A rapid and sensitive method for the quantification of microgram quantities of protein utilizing the principle of protein-dye binding. Anal Biochem 72:248-254

Brown MA, Zhu L, Schmidt C, Tucker PW (2007) Hsp90-from signal transduction to cell transformation. Biochem Biophys Res Commun 363:241-246

Byun S, Lee KW, Jung SK, Lee EJ, Hwang MK, Lim SH, Bode AM, Lee HJ, Dong Z (2010) Luteolin inhibits protein kinase C(epsilon) and c-Src activities and UVB-induced skin cancer. Cancer Res 70:2415-2423

Cai J, Kirlin WG, Chen Y, Yan X, Jones DP, Sartorelli AC (2006) Overexpression of heat shock factor 1 inhibits butyrate-induced differentiation in colon cancer cells. Cell Stress Chaperones 11:199-207

Chen G, Gharib TG, Huang CC et al (2002) Discordant protein and mRNA expression in lung adenocarcinomas. Mol Cell Proteomics 1:304-313

Cho YM, Bae SH, Choi BK, Cho SY, Song CW, Yoo JK, Paik YK (2003) Differential expression of the liver proteome in senescence accelerated mice. Proteomics 3:1883-1894

Christofk HR, Vander Heiden MG, Harris MH, Ramanathan A, Gerszten RE, Wei R, Fleming MD, Schreiber SL, Cantley LC (2008a) The M2 splice isoform of pyruvate kinase is important for cancer metabolism and tumour growth. Nature 452:230 233

Christofk HR, Vander Heiden MG, Wu N, Asara JM, Cantley LC (2008b) Pyruvate kinase M2 is a phosphotyrosine-binding protein. Nature 452:181-186

Decaux JF, Antoine B, Kahn A (1989) Regulation of the expression of the L-type pyruvate kinase gene in adult rat hepatocytes in primary culture. J Biol Chem 264:11584-11590

Diehl MC, Idowu MO, Kimmelshue K, York TP, Elmore LW, Holt SE (2009) Elevated expression of nuclear Hsp90 in invasive breast tumors. Cancer Biol Ther 8:1952-1961 
Elmore LW, Forsythe R, Forsythe H, Bright AT, Nasim S, Endo K, Holt SE (2008) Overexpression of telomerase-associated chaperone proteins in prostatic intraepithelial neoplasia and carcinomas. Oncol Rep 20:613-617

Fleige S, Walf V, Huch S, Prgomet C, Sehm J, Pfaffl MW (2006) Comparison of relative mRNA quantification models and the impact of RNA integrity in quantitative real-time RT-PCR. Biotechnol Lett 28:1601-1613

Fleming SE, Gill R (1997) Aging stimulates fatty acid oxidation in rat colonocytes but does not influence the response to dietary fiber. J Gerontol A Biol Sci Med Sci 52:B318-B330

Fridrich D, Teller N, Esselen M, Pahlke G, Marko D (2008) Comparison of delphinidin, quercetin and (-)-epigallocatechin3-gallate as inhibitors of the EGFR and the ErbB2 receptor phosphorylation. Mol Nutr Food Res 52:815-822

Greiner EF, Guppy M, Brand K (1994) Glucose is essential for proliferation and the glycolytic enzyme induction that provokes a transition to glycolytic energy production. J Biol Chem 269:31484-31490

Guo W, Zhang Y, Chen T, Wang Y, Xue J, Zhang Y, Xiao W, Mo X, Lu Y (2011) Efficacy of RNAi targeting of pyruvate kinase M2 combined with cisplatin in a lung cancer model. J Cancer Res Clin Oncol 137:65-72

Hamer HM, Jonkers D, Venema K, Vanhoutvin S, Troost FJ, Brummer RJ (2008) Review article: the role of butyrate on colonic function. Aliment Pharmacol Ther 27:104-119

Hanahan D, Weinberg RA (2011) Hallmarks of cancer: the next generation. Cell 144:646-674

Hirsch CL, Smith-Windsor EL, Bonham K (2006) Src family kinase members have a common response to histone deacetylase inhibitors in human colon cancer cells. Int J Cancer 118:547-554

Hitosugi $\mathrm{T}$ et al (2009) Tyrosine phosphorylation inhibits PKM2 to promote the Warburg effect and tumor growth. Sci Signal 2:ra73

Hofmann C, Obermeier F, Artinger M, Hausmann M, Falk W, Schoelmerich J, Rogler G, Grossmann J (2007) Cell-cell contacts prevent anoikis in primary human colonic epithelial cells. Gastroenterology 132:587-600

Jahns F, Wilhelm A, Jablonowski N, Mothes H, Radeva M, Wolfert A, Greulich KO, Glei M (2011) Butyrate suppresses mRNA increase of osteopontin and cyclooxygenase-2 in human colon tumor tissue. Carcinogenesis 32:913-920

Jones GM, Mayer RJ (1973) Degradation of glucose-metabolizing enzymes in the rat small intestine during starvation. Biochem $\mathrm{J}$ 132:657-661

Kekatpure VD, Dannenberg AJ, Subbaramaiah K (2009) HDAC6 modulates Hsp90 chaperone activity and regulates activation of aryl hydrocarbon receptor signaling. J Biol Chem 284:7436-7445

Kim YS, Milner JA (2011) Bioactive food components and cancerspecific metabonomic profiles. J Biomed Biotechnol 2011:721213

Koss K, Maxton D, Jankowski JA (2008) Faecal dimeric M2 pyruvate kinase in colorectal cancer and polyps correlates with tumour staging and surgical intervention. Colorectal Dis 10:244-248

Lee HM, Greeley GH Jr, Englander EW (2001) Age-associated changes in gene expression patterns in the duodenum and colon of rats. Mech Ageing Dev 122:355-371

Lin K, Rockliffe N, Johnson GG, Sherrington PD, Pettitt AR (2008) Hsp90 inhibition has opposing effects on wild-type and mutant p53 and induces p21 expression and cytotoxicity irrespective of p53/ATM status in chronic lymphocytic leukaemia cells. Oncogene 27:2445-2455

Marks PA, Xu WS (2009) Histone deacetylase inhibitors: potential in cancer therapy. J Cell Biochem 107:600-608

Mazurek S, Boschek CB, Hugo F, Eigenbrodt E (2005) Pyruvate kinase type $\mathrm{M} 2$ and its role in tumor growth and spreading. Semin Cancer Biol 15:300-308
Milicevic Z, Bogojevic D, Mihailovic M, Petrovic M, Krivokapic Z (2008) Molecular characterization of hsp90 isoforms in colorectal cancer cells and its association with tumour progression. Int $\mathbf{J}$ Oncol 32:1169-1178

Moser C, Lang SA, Kainz S, Gaumann A, Fichtner-Feigl S, Koehl GE, Schlitt HJ, Geissler EK, Stoeltzing O (2007) Blocking heat shock protein-90 inhibits the invasive properties and hepatic growth of human colon cancer cells and improves the efficacy of oxaliplatin in p53-deficient colon cancer tumors in vivo. Mol Cancer Ther 6:2868-2878

Neckers L (2007) Heat shock protein 90: the cancer chaperone. J Biosci 32:517-530

O'Connell K, Gannon J, Doran P, Ohlendieck K (2007) Proteomic profiling reveals a severely perturbed protein expression pattern in aged skeletal muscle. Int J Mol Med 20:145-153

Pearl LH, Prodromou C, Workman P (2008) The Hsp90 molecular chaperone: an open and shut case for treatment. Biochem $\mathrm{J}$ 410:439-453

Peipins LA, Sandler RS (1994) Epidemiology of colorectal adenomas. Epidemiol Rev 16:273-297

Pfaffl MW (2001) A new mathematical model for relative quantification in real-time RT-PCR. Nucleic Acids Res 29:e45

Picard D (2011) HSP90 interactors. http://www.picard.ch/downloads/ Hsp90interactors.pdf. Accessed 16 June 2011

Poole GP, Bloxham DP (1982) The turnover of L-type pyruvate kinase in cultured rat hepatocytes. Biochem J 204:89-95

Pool-Zobel BL, Selvaraju V, Sauer J, Kautenburger T, Kiefer J, Richter KK, Soom M, Wolfl S (2005) Butyrate may enhance toxicological defence in primary, adenoma and tumor human colon cells by favourably modulating expression of glutathione $\mathrm{S}$-transferases genes, an approach in nutrigenomics. Carcinogenesis 26:1064-1076

Radeva M (2009) Expression analysis of a selected gene set in malignant and non-malignant tissues derived from individuals with colon cancer. Comparison with protein expression data. Dissertation, University of Jena

Rahmani M, Yu C, Dai Y, Reese E, Ahmed W, Dent P, Grant S (2003) Coadministration of the heat shock protein 90 antagonist 17-allylamino- 17-demethoxygeldanamycin with suberoylanilide hydroxamic acid or sodium butyrate synergistically induces apoptosis in human leukemia cells. Cancer Res 63:8420-8427

Rashmi R, Santhosh Kumar TR, Karunagaran D (2003) Human colon cancer cells differ in their sensitivity to curcumin-induced apoptosis and heat shock protects them by inhibiting the release of apoptosis-inducing factor and caspases. FEBS Lett 538:19-24

Rogler G, Daig R, Aschenbrenner E, Vogl D, Schlottmann K, Falk W, Gross V, Schölmerich J, Andus T (1998) Establishment of longterm primary cultures of human small and large intestinal epithelial cells. Lab Invest 78:889-890

Sauer J, Richter KK, Pool-Zobel BL (2007) Products formed during fermentation of the prebiotic inulin with human gut flora enhance expression of biotransformation genes in human primary colon cells. Br J Nutr 97:928-937

Scharlau D, Borowicki A, Habermann N, Hofmann T, Klenow S, Miene C, Munjal U, Stein K, Glei M (2009) Mechanisms of primary cancer prevention by butyrate and other products formed during gut flora-mediated fermentation of dietary fibre. Mutat Res 682:39-53

Spoden GA, Mazurek S, Morandell D, Bacher N, Ausserlechner MJ, Jansen-Dürr P, Eigenbrodt E, Zwerschke W (2008) Isotypespecific inhibitors of the glycolytic key regulator pyruvate kinase subtype M2 moderately decelerate tumor cell proliferation. Int J Cancer 123:312-321

Thangaraju M, Carswell KN, Prasad PD, Ganapathy V (2009) Colon cancer cells maintain low levels of pyruvate to avoid cell death 
caused by inhibition of HDAC1/HDAC3. Biochem J 417:379-389

Tran PL, Kim SA, Choi HS, Yoon JH, Ahn SG (2010) Epigallocatechin-3-gallate suppresses the expression of HSP70 and HSP90 and exhibits anti-tumor activity in vitro and in vivo. BMC Cancer 10:276

Traxinger RR, Marshall S (1992) Insulin regulation of pyruvate kinase activity in isolated adipocytes. Crucial role of glucose and the hexosamine biosynthesis pathway in the expression of insulin action. J Biol Chem 267:9718-9723

Trepel J, Mollapour M, Giaccone G, Neckers L (2010) Targeting the dynamic HSP90 complex in cancer. Nat Rev Cancer 10:537-549

Vandesompele J, De PK, Pattyn F, Poppe B, Van RN, De PA, Speleman F (2002) Accurate normalization of real-time quantitative RT-PCR data by geometric averaging of multiple internal control genes. Genome Biol 3:RESEARCH0034

Wolever TM, Chiasson JL (2000) Acarbose raises serum butyrate in human subjects with impaired glucose tolerance. Br J Nutr $84: 57-61$
Wong JM, de Souza R, Kendall CW, Emam A, Jenkins DJ (2006) Colonic health: fermentation and short chain fatty acids. J Clin Gastroenterol 40:235-243

Yamada K, Noguchi T (1999) Nutrient and hormonal regulation of pyruvate kinase gene expression. Biochem J 337(Pt 1):1-11

Yin Z, Henry EC, Gasiewicz TA (2009) (-)-Epigallocatechin-3gallate is a novel Hsp90 inhibitor. Biochemistry 48:336-345

Zhang Y, Zhou L, Bao YL, Wu Y, Yu CL, Huang YX, Sun Y, Zheng LH, Li YX (2010) Butyrate induces cell apoptosis through activation of JNK MAP kinase pathway in human colon cancer RKO cells. Chem Biol Interact 185:174-181

Zwerschke W, Mazurek S, Massimi P, Banks L, Eigenbrodt E, Jansen-Durr P (1999) Modulation of type M2 pyruvate kinase activity by the human papillomavirus type 16 E7 oncoprotein. Proc Natl Acad Sci USA 96:1291-1296

Zwerschke W, Mazurek S, Stockl P, Hutter E, Eigenbrodt E, JansenDurr P (2003) Metabolic analysis of senescent human fibroblasts reveals a role for AMP in cellular senescence. Biochem $\mathbf{J}$ $376: 403-411$ 